\title{
Status of the PANDA experiment at FAIR
}

\author{
A.N. Vasiliev ${ }^{1}$ on behalf of the PANDA Collaboration \\ IHEP \\ Pobeda street 1, Protvino, 142281, Russian Federation \\ E-mail: Alexander.Vasiliev@ihep.ru
}

PANDA (anti-Proton ANnihilations at DArmstadt) is a next generation hadron physics detector planned to be operated at the future Facility for Antiproton and Ion Research (FAIR) at Darmstadt, Germany. It will use intensive cooled antiproton beams with a momentum between $1.5 \mathrm{GeV} / \mathrm{c}$ and $15 \mathrm{GeV} / \mathrm{c}$ interacting with various internal targets. The PANDA detector is a state-of-the-art internal target detector at the High Energy Storage Ring (HESR) at FAIR allowing the detection and identification of neutral and charged particles almost in the whole solid angle. The PANDA physics program and the experimental set-up as well as the current status of the experiment are described in this article.

8th International Conference on Nuclear Physics at Storage Rings (Stori11) Laboratori Nazionali di Frascati dell'INFN, Italy

October 9-14, 2011

1 Speaker 


\section{Introduction}

The modern theory of the strong interactions Quantum Chromodynamics (QCD) is well tested at high energies, where the strong coupling constant becomes small and perturbation theory applies. However, in the low-energy regime QCD becomes a strongly-coupled theory, many aspects of which are not understood. Many questions still exist for this so-called confinement region. Parts of them are as follows: how can we bring order into the rich phenomena of low energy QCD? Are there effective degrees of freedoms in terms of which we can understand the resonances and bound states of QCD efficiently and systematically? Does QCD generate exotic structures, like glueballs, hybrids, etc. so far undiscovered? QCD does not forbid the existence of many exotic states. Obviously new experimental data in this very difficult, - for theory, kinematical region are badly needed. PANDA will be in a unique position to provide answers to such important questions about non-perturbative QCD. A major part of the physics program of PANDA is designed to collect high-quality data that allow a clean interpretation in terms of the predictions of non-perturbative QCD.

The HESR (High Energy Storage Ring) of the new FAIR (Facility for Antiproton and Ion Research) project provides a pure antiproton beam of high resolution (down to $\sigma_{\mathrm{p}} / \mathrm{p}=1 \times 10^{-5}$ with electron cooling available), high intensity (up to $10^{11}$ antiprotons in HESR), in the momentum range from $1.5 \mathrm{GeV} / \mathrm{c}$ to $15 \mathrm{GeV} / \mathrm{c}$. This offers the unique possibility of investigating a broad field of physics phenomena.

\section{PANDA Collaboration}

At present, the PANDA Collaboration consists of more than 460 physicists over the world. See details in figure 1.

\section{Physics program of PANDA}

The PANDA experiment will make the antiproton beam from the HESR colliding with an internal proton target and will use a general purpose spectrometer to carry out a rich and diversified hadron physics program. The experiment is being designed to fully exploit the extraordinary physics potential arising from the availability of high-intensity, cooled antiproton beams. The aim of the rich experimental program is to improve our knowledge of the strong interaction and of hadron structure. Significant progress beyond the present understanding of the field is expected thanks to improvements in statistics and precision of the data with respect to past and present similar programs.

Many experimental goals are foreseen in PANDA. In this article I will mention only a few of them. The QCD bound states are of fundamental importance for a better, quantitative understanding of QCD. Particle's spectra can be computed within the framework of nonrelativistic potential models, effective field theories and Lattice QCD. Therefore, precision measurements are needed to distinguish between the different approaches and identify the relevant degrees of freedom. The measurements to be carried out in PANDA include charmonium, D meson and baryon spectroscopy. Charmonium will be mainly extensively 
studied above the D-Dbar threshold $(3.74 \mathrm{GeV})$, where many new states are expected by Lattice QCD and some other models. However some precise measurements, like $h_{c}$ width and more, below this threshold are still relevant. In addition, PANDA will look for exotic states such as gluonic hadrons (hybrids and glueballs), multi-quark and molecular states. We test the binding of quarks to hadrons by exciting the bound states in all known ways, rotation (high radial excitation), stretching the glue string (high orbit), vibration of the glue string (hybrids). Potential of non-relativistic quarks is well verified by lattice QCD. Decorated with typical terms which are well known from other bound states (hydrogen, positronium), one obtains a spectrum which is motivated by one gluon exchange. The gluon may also vibrate and thus contributes to the quantum numbers of the state. Then we have in addition to the simple $\Sigma$, also $\pi^{-}$and many other potentials. Hybrids are bound states of these higher potentials.

\section{- At present a group of $\mathbf{4 6 0}$ physicists from 55 institutions of 17 countries

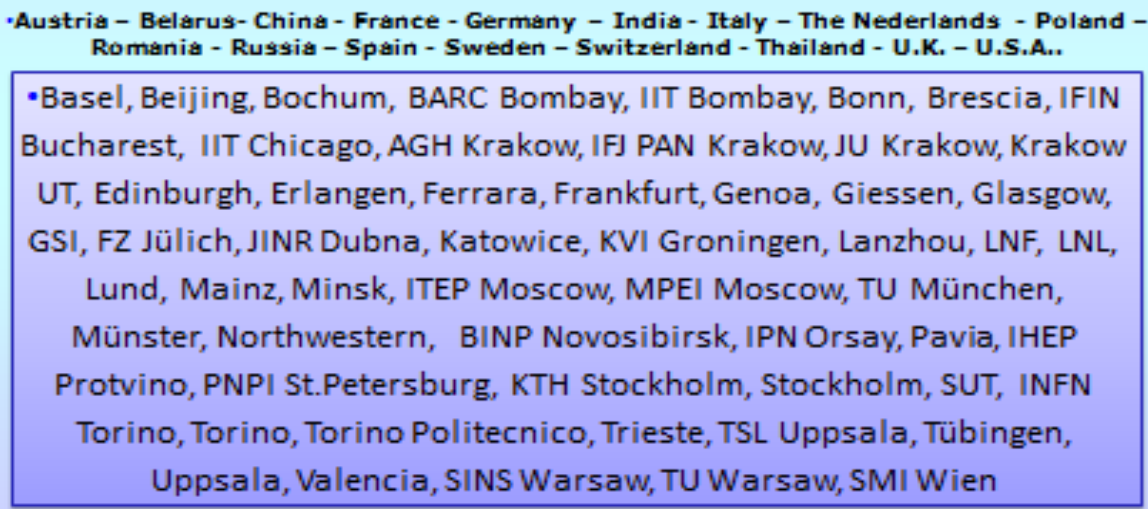

Figure 1: The PANDA Collaboration.

The study of medium modifications of hadrons's properties - when embedded in hadronic matter - is aimed at understanding the origin of hadron's masses in the context of spontaneous chiral symmetry breaking in QCD and its partial restoration in a hadronic environment. So far experiments have been focused on the light quark sector. The high-intensity antiproton beam up to $15 \mathrm{GeV} / \mathrm{c}$ momentum will allow an extension of this program to the charm sector both for hadrons with hidden and open charm. The in-medium masses of these states are expected to be affected primarily by the gluon condensate.

Hyper-nuclei are systems in which up or down quarks are replaced by strange quarks. In this way a new quantum number, strangeness, is introduced into the nucleus. This open new perspectives for nuclear structure spectroscopy and for studying the forces between hyperons and nucleons.

PANDA will be able to investigate the structure of the nucleon using electromagnetic processes, such as Deeply Virtual Compton Scattering and the process antiproton+proton $\rightarrow \mathrm{e}^{+} \mathrm{e}^{-}$, which will allow the determination of the electromagnetic form-factors of the proton in the time-like region over an extended $\mathrm{q}^{2}$ region. The PANDA physics program is described in details in [1]. 
In antiproton-proton annihilations, there is one main advantage compared to $\mathrm{e}^{+} \mathrm{e}^{-}$annihilation: any resonances can be formed directly, because there are no restrictions for the quantum numbers of the final state. In contrast, in the $\mathrm{e}^{+} \mathrm{e}^{-}$- annihilation only $\mathrm{J}^{\mathrm{PC}}=1^{--}$states, like for example the $\mathrm{J} / \psi$-particle can be produced. There is one more big advantage of PANDA at HESR. The study of resonances is an important part of the PANDA physics program. Masses, widths and decay fractions even for narrow resonances will be measured by scanning the beam energy across the resonance under study with a precise accuracy. At the first stage of PANDA (the so called FAIR Modularized Start Version) due to stochastic cooling, $\sigma_{\mathrm{p}} / \mathrm{p}$ for antiprotons is anticipated at the level of $10^{-4}$. Therefore, the widths of resonances sort of a few tens of $\mathrm{MeV}$ could be measured precisely with a scan step of $1 \mathrm{MeV}$ or so. Later on, when electron cooling will become available, $\sigma_{\mathrm{p}} / \mathrm{p}$ for antiprotons will be decreased by several times, and consequently the widths of resonances sort of several $\mathrm{MeV}$ could be measured with an unprecedented accuracy.

\section{PANDA experimental set-up}

PANDA is one of the experiments at the new FAIR facility (see figure 2), which is being built in Darmstadt, Germany, beside GSI.

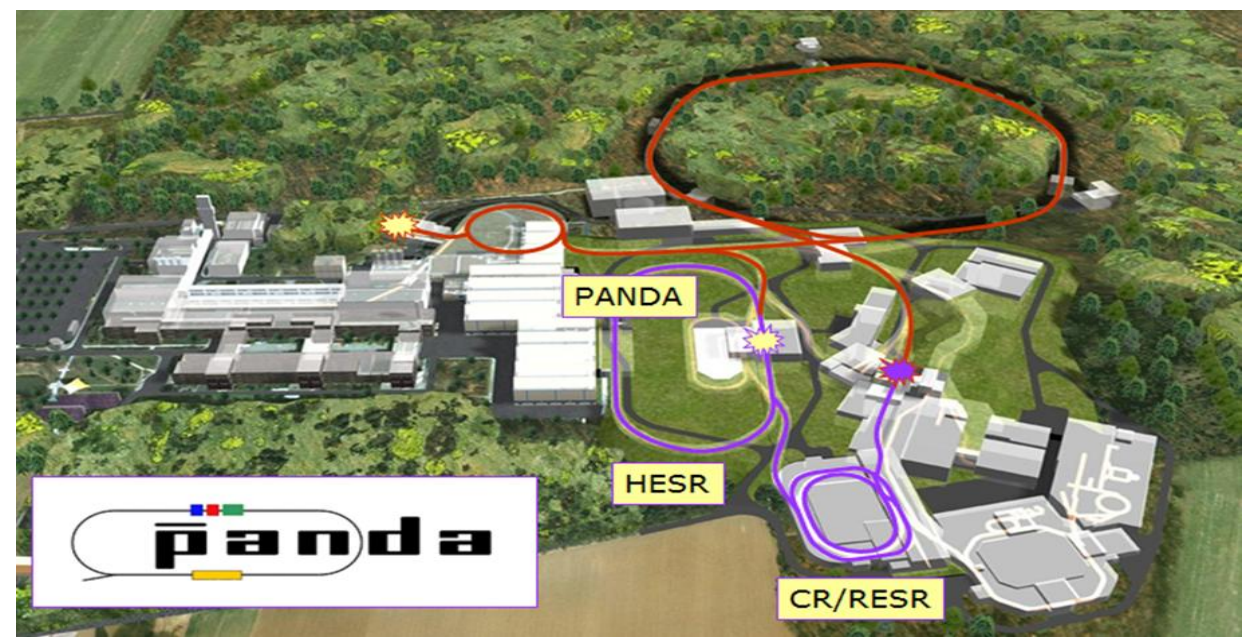

Figure 2: Schematic view of the new FAIR facility in Darmstadt (Germany).

The main objectives of the design of the PANDA experiment, pictured in figure 3 , are to achieve $4 \pi$ - acceptance, high resolution for tracking, particle identification and calorimetry, high rate capabilities and a versatile readout and event selection. To obtain a good momentum resolution the detector is split into a target spectrometer based on a superconducting solenoid magnet surrounding the interaction point and measuring at high angles and a forward spectrometer based on a dipole magnet for small angle tracks. A silicon vertex detector surrounds the interaction point. In both spectrometer parts, charged particle tracking and identification, electromagnetic calorimetry and muon identification are available to allow detecting the complete spectrum of final states relevant for the PANDA physics objectives.

The target spectrometer surrounds the interaction point and measures charged tracks in a solenoidal field of $2 \mathrm{~T}$. It consists of detector layers arranged in an onion shell configuration. 
Pipes for the injection of target material have to cross the spectrometer perpendicular to the beam pipe. The target spectrometer is arranged in a barrel part for angles larger than $22^{\circ}$ and an end-cap part for the forward range down to $5^{0}$ in the vertical and $10^{0}$ in the horizontal plane. One of the main design requirements is compactness to avoid a too large and a too costly magnet and crystal electromagnetic calorimeter.

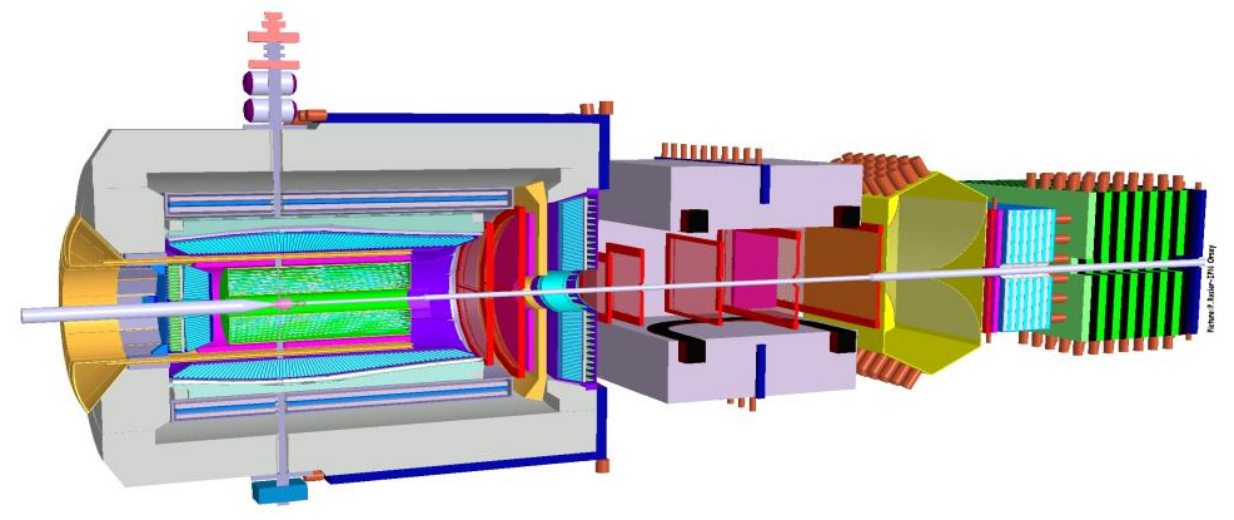

Figure 3. The PANDA experimental set-up. An antiproton beam will go from the left to the right within the vacuum tube of the HESR accelerator through the set-up. The overall length is about $12 \mathrm{~m}$. The height of the solenoid (on the left) is about 5 meters. Other details are in the text.

In order to reach the design luminosity of $2 \cdot 10^{32} \mathrm{~cm}^{-2} \mathrm{~s}^{-1}$ a target thickness of about $4 \cdot 10^{15}$ hydrogen atoms per $\mathrm{cm}^{2}$ is required assuming $10^{11}$ stored anti-protons in the HESR ring. These are conditions posing a real challenge for an internal target inside a storage ring. At present, two different, complementary techniques for the internal target are being developed: the clusterjet target and the pellet target. Both techniques are capable of providing sufficient densities for hydrogen at the interaction point, but exhibit different properties concerning their effect on the beam quality and the definition of the interaction point. A cluster-jet with density up to $10^{15}$ atoms $/ \mathrm{cm}^{2}$ is expected to be in PANDA. A pellet target is anticipated to have an average intensity of $4 \cdot 10^{15}$ hydrogen atoms per $\mathrm{cm}^{2}$. The advantage of the cluster-jet target is that the density can be varied continuously, allowing to have a constant event rate. The disadvantage is in the weak definition of the interaction point compared to the pellet target, where this interaction point is being defined precisely. A big work still has to be done to minimize immediate event rate jumps in the interactions with the pellet target, followed by sharp decreasing of event rate in the intervals between the pellets.

The magnetic field in the target spectrometer is provided by a superconducting solenoid [2] whose coil has an inner radius of $90 \mathrm{~cm}$ and a length of $2.8 \mathrm{~m}$. The maximum magnetic field is $2 \mathrm{~T}$. The field homogeneity is foreseen to be better than $2 \%$ over the volume of the vertex detector and the central tracker.

The design of the micro-vertex detector (MVD) for the target spectrometer is optimized for the detection of secondary vertices from D-mesons and hyperon decays and the maximum acceptance close to the interaction point. It will also strongly improve the transverse momentum resolution. The present design of the pixel detectors comprises silicon sensors, which are 100 $\mu \mathrm{m}$ thick $\left(0.1 \% \mathrm{X}_{0}\right)$. 
The charged particle tracking devices must handle the high particle fluxes that are anticipated for a luminosity of up to several $10^{32} \mathrm{~cm}^{-2} \mathrm{~s}^{-1}$. The momentum resolution $\sigma_{\mathrm{p}} / \mathrm{p}$ has to be on the percent level. The detectors should have good detection efficiency for secondary vertices which can occur outside the inner vertex detector (e.g. $\mathrm{K}_{\mathrm{S}}^{0}$ or $\Lambda$ ). This is achieved by the combination of the MVD close to the interaction point with two outer systems. One is covering a large area and is designed as a barrel around the MVD. This will be a stack of straw tubes and is called straw tubes tracker (STT). The forward angles will be covered using three sets of GEM trackers. The STT consists of aluminized mylar straw tubes, which are selves supporting by the operation at 1 bar overpressure. The straws are arranged in planar layers which are mounted in a hexagonal shape around the MVD. In total there are 27 layers of which the 8 central ones are tilted to achieve an acceptable resolution of $3 \mathrm{~mm}$ also in $\mathrm{z}$ (parallel to the beam), while the resolution in $\mathrm{x}$ and $\mathrm{y}$ directions is anticipated to be $150 \mu \mathrm{m}$. The gap to the surrounding detectors is filled with further individual straws. In total there are 4600 straws around the beam pipe at radial distances between $15 \mathrm{~cm}$ and $42 \mathrm{~cm}$ with an overall length of 150 $\mathrm{cm}$. All straws have a diameter of $1 \mathrm{~cm}$.

Charged particle identification of hadrons and leptons over a large range of angles and momenta is an essential requirement for meeting the physics objectives of PANDA. The main part of the momentum spectrum above $1 \mathrm{GeV} / \mathrm{c}$ will be covered by Cherenkov detectors. Below the Cherenkov threshold of kaons a time-of-flight detector can identify slow particles. EMC can identify electrons. Charged particle identification in the target spectrometer will be performed by the detection of internally reflected Cherenkov (DIRC) light, while in the forward region there is a forward endcap DIRC. The radiator material chosen for these detectors is silica (i.e. artificial quartz), with a refractive index of 1.47. This provides pion-kaon separation from rather low momenta of $800 \mathrm{MeV} / \mathrm{c}$ up to about $5 \mathrm{GeV} / \mathrm{c}$ and fits well to the compact design of the target spectrometer. For slow particles at large and small polar angles particle identification will be provided by time-of-flight (TOF) detectors. Both, the barrel (pads) and the forward TOF (bars), will be scintillator detectors.

The expected high count rates and a geometrically compact design of the target spectrometer require a fast scintillator material with a short radiation length and a small Moliere radius for the construction of the electromagnetic calorimeter (EMC). Lead tungstate $\left(\mathrm{PbWO}_{4}\right)$ is a high density inorganic scintillator with sufficient energy and time resolution for photon, electron, and hadron detection even at intermediate energies [3]. Recent developments indicate a significant increase of light yield due to crystal perfectioning and appropriate doping to enable photon detection down to a few $\mathrm{MeV}$ with sufficient resolution. The light yield can be increased by a factor of about 4 compared to room temperature by cooling the crystals down to $25^{0} \mathrm{C}$. The crystals of the PANDA EMC will be $20 \mathrm{~cm}$ long, i.e. approximately $22 \mathrm{X}_{0}$, in order to achieve an energy resolution below $2 \%$ at $1 \mathrm{GeV}$ and a tolerable energy loss due to longitudinal leakage of the shower. Tapered crystals with a front size of $2.1 \cdot 2.1 \mathrm{~cm}^{2}$ will be mounted at an inner radius of $57 \mathrm{~cm}$. This implies 11360 crystals for the barrel part of the calorimeter. The forward end-cap calorimeter will have 3600 tapered crystals, the backward end-cap calorimeter 592. The readout of the crystals will be accomplished by large area 
avalanche photo diodes in the barrel and vacuum photo-triodes in the forward and backward end caps.

Muons are an important probe for, among others, $\mathrm{J} / \psi$ decays, semi-leptonic D-meson decays and the Drell-Yan process. The majority of background particles are pions and their decay daughter muons. However at the low momenta of PANDA the signature is less clean than in high energy physics experiments. To allow nevertheless a proper separation of primary muons from pions and decay muons a range tracking system will be implemented in the yoke of the solenoid magnet. Here a fine segmentation of the yoke as absorber with interleaved tracking detectors allows the distinction of energy loss processes of muons and pions and kinks from pion decays.

A dipole magnet [2] with a window frame, a $1 \mathrm{~m}$ gap, and more than $2 \mathrm{~m}$ aperture will be used for the momentum analysis of charged particles in the forward spectrometer. In the current planning, the magnet yoke will occupy about $2.5 \mathrm{~m}$ in beam direction starting from $3.5 \mathrm{~m}$ downstream of the target. Thus, it covers the entire angular acceptance of the target spectrometer of $\pm 10^{0}$ and $\pm 5^{\circ}$ in the horizontal and in the vertical directions, respectively. The maximum bending power of the magnet will be $2 \mathrm{Tm}$.

The deflection of particle trajectories in the field of the dipole magnet will be measured with a set of straw tubes of $1 \mathrm{~cm}$ diameter, two stations placed in front, two within and two behind the dipole magnet. A wall of slabs made of plastic scintillator and read out on both ends by fast photo tubes will serve as time-of-flight stop counter placed at about $7 \mathrm{~m}$ from the target. In addition, similar detectors will be placed inside the dipole magnet opening, to detect low momentum particles which do not exit the dipole magnet. For the detection of photons and electrons a Shashlyk-type calorimeter with high resolution and efficiency will be employed. The detection is based on lead-scintillator sandwiches read out with wavelength shifting fibers passing through the block and coupled to photomultipliers. To cover the forward acceptance, 26 rows and 54 columns are required with a cell size of $55 \mathrm{~mm}$, i.e. 1404 modules in total, which will be placed at a distance of 7-8 m from the target. For the very forward part of the muon spectrum a further range tracking system is being designed.

\section{Schedule}

The schedule to build up the PANDA set-up and then commission it and start the data taking looks as follows. The Technical Design Reports (TDR) for the majority of the subdetectors and systems of PANDA will have to be ready by the end of 2012. Most of the R\&D works must be finished by the end of 2012, except that related to the pellet target and the barrel TOF. Up to now we have already purchased about 9,000 lead tungstate crystals out of 16,000 ones for the target EMC, therefore we can consider that we have already started mass production. We will have to start the remaining mass production since 2013. We plan to have pre-assembling of some parts of the PANDA set-up at Juelich since 2015. The transition of the equipment from Juelich, Germany to Darmstadt is anticipated to be in 2017. Then we will immediately start to assemble the whole PANDA set-up at the HESR working place of PANDA. We plan to have commissioning of HESR in 2017 and commissioning of the PANDA set-up in 2018. Soon afterwards we expect to have first data taking. 


\section{Conclusion}

To study fundamental questions of hadron and nuclear physics in interactions of antiprotons with nucleons and nuclei, the unique universal PANDA experiment at FAIR is under preparation. Gluonic excitations, the physics of strange and charm quarks and nucleon structure studies will be performed with world record accuracy thereby, allowing high-precision tests of the strong interaction. The proposed PANDA detector is a state-of-the art internal target detector at the HESR at FAIR allowing the detection and identification of neutral and charged particles generated within the relevant angular and energy ranges. The uniqueness of PANDA is the following: the intensity of pure antiproton beam and the density of the internal target are so that the luminosity will arrive up to $2 \cdot 10^{32} \mathrm{~cm}^{-2} \mathrm{~s}^{-1}$ at some advanced stage of the experiment. By using stochastic cooling in the frame of the Modularized Start Version of FAIR, $\sigma_{\mathrm{p}} / \mathrm{p}$ of the antiproton beam is anticipated at the level of $10^{-4}$. Later, when the electron cooling will become available, the value of $\sigma_{\mathrm{p}} / \mathrm{p}$ will decrease of several times. Consequently, in the scan mode of the PANDA experiment with a pretty small scan step, the parameters of the new states, like mass, width, branching ratios, might be measured with unprecedented accuracy. The momentum of the antiproton beam can be within the $1.5-15 \mathrm{GeV} / \mathrm{c}$.

The hadron physics program of PANDA does not permit a protracted staging for setting up the detector. To carry out any of the physics goals approved for PANDA, the two magnetic (target and forward) spectrometers with charged particle tracking, a high-resolution electromagnetic calorimeter and particle ID in $4 \pi$ are mandatory. This constitutes the core of the PANDA detector and must be set up in the first stage of the experiment in the FAIR Modularized Start Version. For example, a thorough study of recently discovered new and unexpected states of matter is not feasible with a detector that lacks of any of the above features. The PANDA data acquisition system should work effectively at $2 \cdot 10^{7}$ interactions per second.

The search for new forms of matter in the interaction of antimatter with matter in the PANDA experiment using high-intensity monochromatic beam of antiprotons is a unique addition to the ultrahigh-energy physics of the LHC. Nowhere in the world a similar experimental project is being planned, because there is no corresponding experimental basis for these basic research.

About 460 physicists from 17 countries in Europe, Asia and Northern America are involved in the PANDA project. Commissioning the PANDA experimental set-up and first data taking are anticipated in 2018.

\section{References}

[1] PANDA Collaboration, Physics Performance Report for PANDA: Strong Interaction Studies with Antiprotons, hep-ex/0903.3905

[2] PANDA Collaboration, Technical Design Report for the PANDA Solenoid and Dipole Spectrometer Magnets, hep-ex/0907.0169.

[3] PANDA Collaboration, Design Report for PANDA Electromagnetic Calorimeter (EMC), hepex/0810.1216 